\title{
The Representation of Matrices in Unit Vector Notation
}

\author{
Pallavi Sudhir Abhimanyu ${ }^{1}$ \\ ${ }^{1}$ NUS High School of Mathematics and Science, Clementi, Singapore \\ Correspondence: Pallavi Sudhir Abhimanyu, NUS High School of Mathematics and Science, Clementi, Singapore. \\ Tel: 65-9640-7571. E-mail: h1210084@nus.edu.sg
}

Received: June 17, 2012 Accepted: July 4, 2012 Online Published: July 20, 2012

doi:10.5539/jmr.v4n4p86 URL: http://dx.doi.org/10.5539/jmr.v4n4p86

\begin{abstract}
The objective of this paper is to express a matrix of any dimension in unit vector notation. This is accomplished by first solving the two and three dimensional cases before solving the general $\mathrm{n}$-dimensional case. The fact that matrices can be represented as a (non-linear) combination of standard basis unit vectors shows that matrices are not simply abstract entities used just for representing data, but also have a geometric interpretation. This paper then defines the natural product and explains its applications in matrix calculus, before relating it to the outer product of real vectors, a special case of the tensor product. We then conclude that the space is the intersection of the exterior space and the newly defined natural space.
\end{abstract}

Keywords: linear algebra, clifford algebra, matrices in unit vector notation, matrix calculus, outer product

\section{Introduction}

Representing vectors in unit vector notation evolved from Sir William Rowan Hamiltons discovery of quaternions, which he published in various books, one of which was the Elements of Quaternions (Hamilton, 1866). The unit quaternions, $i, j$ and $k$ act as a basis for all quaternions. However, at the turn of the 20th century, vector analysis began to gain prominence, through works by various mathematicians including Gibbs (1913). Gradually, the work which used to be represented in terms of quaternions began being represented in terms of unit vectors of the standard basis. Initially, they were represented using $\hat{\imath}, \hat{\jmath}, \hat{k}$ but soon, in the realms of Mathematics and Theoretical Physics, the notation $\hat{\mathbf{e}}_{1}, \hat{\mathbf{e}}_{2}, \hat{\mathbf{e}}_{3}, \ldots$ was adopted, since it is consistent with any dimension, whereas the $\hat{\imath}, \hat{\jmath}, \hat{k}$ notation was retained in Engineering, where objects are considered in no more than 3 dimensions. Latyshev (2005) generalised the standard basis to any linear space over any field. However, representing matrices in unit vectors never gained prominence among the academic community and has been assumed to be not generally possible. This paper challenges this assumption and makes an attempt to express a matrix of any dimension in unit vector notation. Finding an equation which expresses a matrix of any dimension in unit vector notation is important as it allows a geometric interpretation of matrices and also has applications in matrix calculus as discussed later.

\section{2. $2 \times 2$ Matrices in Unit Vector Notation}

Let matrix $\mathbf{A}$ be defined as follows:

$$
\mathbf{A}=\left[\begin{array}{ll}
\alpha & \beta \\
\gamma & \delta
\end{array}\right]
$$

Taking the transpose,

$$
\mathbf{A}=\left[\begin{array}{ll}
\alpha & \gamma \\
\beta & \delta
\end{array}\right]^{T}
$$

Writing each column in unit vector notation,

$$
\mathbf{A}=\left[\begin{array}{ll}
\alpha \hat{\imath}+\beta \hat{\jmath} & \gamma \hat{\imath}+\delta \hat{\jmath}
\end{array}\right]^{T}
$$

Taking the transpose again,

$$
\mathbf{A}=\left[\begin{array}{l}
\alpha \hat{\imath}+\beta \hat{\jmath} \\
\gamma \hat{\imath}+\delta \hat{\jmath}
\end{array}\right]
$$

Finally,

$$
\mathbf{A}=\alpha \hat{\iota}^{2}+\beta \hat{\jmath}+\gamma \hat{\imath} \hat{\jmath}+\delta \hat{\jmath}^{2}
$$


As required, we have expressed a 2 by 2 matrix as a nonlinear combination of the first two standard basis unit vectors. It is to be noted that $\overrightarrow{\mathbf{a}} \overrightarrow{\mathbf{b}}$ is not the geometric product of the vectors $\overrightarrow{\mathbf{a}}$ and $\overrightarrow{\mathbf{b}}$ here.

\section{3. $3 \times 3$ Matrices in Unit Vector Notation}

Let matrix $\mathbf{B}$ be defined as follows:

$$
\mathbf{B}=\left[\begin{array}{lll}
b_{11} & b_{12} & b_{13} \\
b_{21} & b_{22} & b_{23} \\
b_{31} & b_{32} & b_{33}
\end{array}\right]
$$

Repeating the same process we did in Section 2,

$$
\begin{gathered}
\mathbf{B}=\left[\begin{array}{lll}
b_{11} & b_{21} & b_{31} \\
b_{12} & b_{22} & b_{32} \\
b_{13} & b_{23} & b_{33}
\end{array}\right]^{T} \\
\mathbf{B}=\left[\begin{array}{ll}
b_{11} \hat{\mathbf{e}}_{1}+b_{12} \hat{\mathbf{e}}_{2}+b_{13} \hat{\mathbf{e}}_{3} \quad b_{21} \hat{\mathbf{e}}_{1}+b_{22} \hat{\mathbf{e}}_{2}+b_{23} \hat{\mathbf{e}}_{3} & b_{31} \hat{\mathbf{e}}_{1}+b_{32} \hat{\mathbf{e}}_{2}+b_{33} \hat{\mathbf{e}}_{3}
\end{array}\right]^{T} \\
\mathbf{B}=\left[\begin{array}{l}
b_{11} \hat{\mathbf{e}}_{1}+b_{12} \hat{\mathbf{e}}_{2}+b_{13} \hat{\mathbf{e}}_{3} \\
b_{21} \hat{\mathbf{e}}_{1}+b_{22} \hat{\mathbf{e}}_{2}+b_{23} \hat{\mathbf{e}}_{3} \\
b_{31} \hat{\mathbf{e}}_{1}+b_{32} \hat{\mathbf{e}}_{2}+b_{33} \hat{\mathbf{e}}_{3}
\end{array}\right] \\
\mathbf{B}=b_{11} \hat{\mathbf{e}}_{1}^{2}+b_{12} \hat{\mathbf{e}}_{2} \hat{\mathbf{e}}_{1}+b_{13} \hat{\mathbf{e}}_{3} \hat{\mathbf{e}}_{1}+b_{21} \hat{\mathbf{e}}_{1} \hat{\mathbf{e}}_{2}+b_{22} \hat{\mathbf{e}}_{2}^{2}+b_{23} \hat{\mathbf{e}}_{3} \hat{\mathbf{e}}_{2}+b_{31} \hat{\mathbf{e}}_{1} \hat{\mathbf{e}}_{3}+b_{32} \hat{\mathbf{e}}_{2} \hat{\mathbf{e}}_{3}+b_{33} \hat{\mathbf{e}}_{3}^{2}
\end{gathered}
$$

As required, we have expressed a 3 by 3 matrix as a nonlinear combination of the first three standard basis unit vectors.

\section{4. $4 \times 4$ Matrices in Unit Vector Notation}

Let matrix $\mathbf{C}$ be defined as follows:

$$
\mathbf{C}=\left[\begin{array}{llll}
a & b & c & d \\
e & f & g & h \\
m & n & p & q \\
r & s & t & u
\end{array}\right]
$$

Once again repeating the process in Sections 2 and 3,

$$
\mathbf{C}=\left[\begin{array}{llll}
a & e & m & r \\
b & f & n & s \\
c & g & p & t \\
d & h & q & u
\end{array}\right]^{T}
$$

$$
\mathbf{C}=\left[\begin{array}{llll}
a \hat{\mathbf{e}}_{1}+b \hat{\mathbf{e}}_{2}+c \hat{\mathbf{e}}_{3}+d \hat{\mathbf{e}}_{4} & e \hat{\mathbf{e}}_{1}+f \hat{\mathbf{e}}_{2}+g \hat{\mathbf{e}}_{3}+h \hat{\mathbf{e}}_{4} & m \hat{\mathbf{e}}_{1}+n \hat{\mathbf{e}}_{2}+p \hat{\mathbf{e}}_{3}+q \hat{\mathbf{e}}_{4} & r \hat{\mathbf{e}}_{1}+s \hat{\mathbf{e}}_{2}+t \hat{\mathbf{e}}_{3}+u \hat{\mathbf{e}}_{4}
\end{array}\right]^{T}
$$

$$
\mathbf{C}=\left[\begin{array}{c}
a \hat{\mathbf{e}}_{1}+b \hat{\mathbf{e}}_{2}+c \hat{\mathbf{e}}_{3}+d \hat{\mathbf{e}}_{4} \\
e \hat{\mathbf{e}}_{1}+f \hat{\mathbf{e}}_{2}+g \hat{\mathbf{e}}_{3}+h \hat{\mathbf{e}}_{4} \\
m \hat{\mathbf{e}}_{1}+n \hat{\mathbf{e}}_{2}+p \hat{\mathbf{e}}_{3}+q \hat{\mathbf{e}}_{4} \\
r \hat{\mathbf{e}}_{1}+s \hat{\mathbf{e}}_{2}+t \hat{\mathbf{e}}_{3}+u \hat{\mathbf{e}}_{4}
\end{array}\right]
$$

$\mathbf{C}=a \hat{\mathbf{e}}_{1}^{2}+b \hat{\mathbf{e}}_{2} \hat{\mathbf{e}}_{1}+c \hat{\mathbf{e}}_{3} \hat{\mathbf{e}}_{1}+d \hat{\mathbf{e}}_{4} \hat{\mathbf{e}}_{1}+e \hat{\mathbf{e}}_{1} \hat{\mathbf{e}}_{2}+f \hat{\mathbf{e}}_{2}^{2}+g \hat{\mathbf{e}}_{3} \hat{\mathbf{e}}_{2}+h \hat{\mathbf{e}}_{4} \hat{\mathbf{e}}_{2}+m \hat{\mathbf{e}}_{1} \hat{\mathbf{e}}_{3}+h \hat{\mathbf{e}}_{2} \hat{\mathbf{e}}_{3}+p \hat{\mathbf{e}}_{3}^{2}+q \hat{\mathbf{e}}_{4} \hat{\mathbf{e}}_{3}+r \hat{\mathbf{e}}_{1} \hat{\mathbf{e}}_{4}+s \hat{\mathbf{e}}_{2} \hat{\mathbf{e}}_{4}+t \hat{\mathbf{e}}_{3} \hat{\mathbf{e}}_{4}+u \hat{\mathbf{e}}_{4}^{2}$

As required, we have expressed a 4 by 4 matrix as a nonlinear combination of the first four standard basis unit vectors.

\section{5. $m \times n$ Matrices in Unit Vector Notation}

Let matrix $\mathbf{A}$ be defined as follows:

$$
\underset{m \times n}{\mathbf{A}}=\left[\begin{array}{ccc}
a_{11} & \cdots & a_{1 n} \\
\vdots & \ddots & \vdots \\
a_{m 1} & \cdots & a_{m n}
\end{array}\right]
$$


Repeating the process in Sections 2, 3 and 4,

$$
\begin{gathered}
\mathbf{A}=\left[\begin{array}{ccc}
a_{11} & \ldots & a_{m 1} \\
\vdots & \ddots & \vdots \\
a_{1 n} & \cdots & a_{m n}
\end{array}\right]^{T} \\
\mathbf{A}=\left[\sum_{j=1}^{n} a_{1 j} \hat{\mathbf{e}}_{j} \cdots \sum_{k=1}^{n} a_{m k} \hat{\mathbf{e}}_{k}\right]^{T} \\
\mathbf{A}=\left[\begin{array}{c}
\sum_{j=1}^{n} a_{1 j} \hat{\mathbf{e}}_{j} \\
\vdots \\
\sum_{k=1}^{n} a_{m k} \hat{\mathbf{e}}_{k}
\end{array}\right] \\
\mathbf{A}=\left(\sum_{j=1}^{n} a_{1 j} \hat{\mathbf{e}}_{j}\right) \hat{\mathbf{e}}_{1}+\ldots+\left(\sum_{k=1}^{n} a_{m k} \hat{\mathbf{e}}_{k}\right) \hat{\mathbf{e}}_{m} \\
\mathbf{A}=\sum_{j=1}^{n} a_{1 j} \hat{\mathbf{e}}_{j} \hat{\mathbf{e}}_{1}+\ldots+\sum_{k=1}^{n} a_{m k} \hat{\mathbf{e}}_{k} \hat{\mathbf{e}}_{m} \\
\mathbf{A}=\sum_{k=1}^{m}\left(\sum_{j=1}^{n} a_{k j} \hat{\mathbf{e}}_{j} \hat{\mathbf{e}}_{k}\right)
\end{gathered}
$$

And thus,

$$
\underset{m \times n}{\mathbf{A}}=\left[\begin{array}{ccc}
a_{11} & \ldots & a_{1 n} \\
\vdots & \ddots & \vdots \\
a_{m 1} & \cdots & a_{m n}
\end{array}\right]=\sum_{k=1}^{m}\left(\sum_{j=1}^{n} a_{k j} \hat{\mathbf{e}}_{j} \hat{\mathbf{e}}_{k}\right)
$$

As required.

\section{6. $m \times n \times p$ Matrices in Unit Vector Notation}

First of all, in order to express three dimensional matrices in unit vector notation, we must make the following definition about the transpose of a three dimensional matrix:

If there exist two three dimensional matrices $\mathbf{A}$ and $\mathbf{B}$ such that if $\mathbf{A}=\left[a_{i j k}\right]$ and $\mathbf{B}=\left[b_{\kappa \lambda \mu}\right]$ then $a_{i j k}=b_{j k i}$. Then, $\mathbf{B}$ is said to be the transpose of $\mathbf{A}$ and this is written as $\mathbf{B}=\mathbf{A}^{T m}$

Now, let $\mathbf{B}$ be defined as follows:

$$
\underset{m \times n \times p}{\mathbf{B}}=\left[b_{i j k}\right]
$$

Here, $i, j$ and vary between 1 and $m, 1$ and $n$ and 1 and $p$ respectively. Then, we can manipulate $\mathbf{B}$ as shown below:

$$
\begin{gathered}
\mathbf{B}=\left(\left[b_{j k i}\right]^{T m}\right)^{T m} \\
\mathbf{B}=\left[b_{k i j}\right]^{T m}
\end{gathered}
$$

The matrix $\left[b_{k i j}\right]$ is a $p$ by $m$ by $n$ matrix and its top view is an $m$ by $n$ matrix. So each layer of this matrix parallel to the ground beneath this matrix is an $m$ by $n$ matrix. The $r^{\text {th }}$ layer is the $m$ by $n$ matrix $\left[b_{r i j}\right]$ where $r$ is fixed and $i$ and $j$ vary between 1 and $m$ and 1 and $n$ respectively.

$$
\left[b_{r i j}\right]=\sum_{k=1}^{m} \sum_{j=1}^{n} b_{r k j} \hat{\mathbf{e}}_{j} \hat{\mathbf{e}}_{k}
$$


The matrix $\left[b_{k i j}\right]$ is an array of this expression in the third dimension as $r$ varies from 1 to $p$, i.e.

$$
\underset{m \times n \times p}{\mathbf{B}}=\left[b_{k i j}\right]^{T m}=\left[\begin{array}{l}
\sum_{k=1}^{m} \sum_{j=1}^{n} b_{1 k j} \hat{\mathbf{e}}_{j} \hat{\mathbf{e}}_{k} \\
\sum_{k=1}^{m} \sum_{j=1}^{n} b_{2 k j} \hat{\mathbf{e}}_{j} \hat{\mathbf{e}}_{k} \\
\vdots \\
\sum_{k=1}^{m} \sum_{j=1}^{n} b_{p k j} \hat{\mathbf{e}}_{j} \hat{\mathbf{e}}_{k}
\end{array}\right]
$$

Finally,

$$
\mathbf{B}=\left[b_{i j k}\right]=\sum_{i=1}^{p} \sum_{k=1}^{m} \sum_{j=1}^{n} b_{i k j} \hat{\mathbf{e}}_{j} \hat{\mathbf{e}}_{k} \hat{\mathbf{e}}_{i}
$$

\section{A Formula for the Expression of a Matrix of Any Dimension in Unit Vector Notation}

We must make the following definition about the transpose of a matrix of any dimension: If there exist two $r$ dimensional matrices $\mathbf{A}$ and $\mathbf{B}$ such that if $\mathbf{A}=\left[a_{j_{1} j_{2} j_{3} \ldots j_{r}}\right]$ and $\mathbf{B}=\left[b_{\kappa_{1} \kappa_{2} \kappa_{3} \ldots \kappa_{r}}\right]$, then . Then, $\mathbf{B}$ is said to be the transpose of $\mathbf{A}$ and this is written as $\mathbf{B}=\mathbf{A}^{T m}$.

Then, applying the same method as in Section 6, we obtain:

$$
\underset{p_{1} \times p_{2} \times \ldots \times p_{n}}{\mathbf{A}}=\sum_{x_{1}, x_{2}, x_{3}, \ldots x_{r}}\left(a_{x_{1}, x_{r}, x_{r-1}, \ldots x_{2}}\left(\prod_{n=2}^{r} \hat{\mathbf{e}}_{n}\right) \hat{\mathbf{e}}_{1}\right)
$$

\section{The Natural Product}

We define the natural product as a mapping which satisfies the following properties:

1) The natural product is associative.

2) The natural product is distributive.

Here, $\mathbb{M}$ represents the set of all matrices of any dimension and $\times$ represents the Cartesian Product. The specific type of natural product $\hat{\mathbf{e}}_{m \wp} \wp \hat{\mathbf{e}}_{n}$ is represented simply as $\hat{\mathbf{e}}_{m} \hat{\mathbf{e}}_{n}$. In the previous sections, $\hat{\mathbf{e}}_{n_{1}} \hat{\mathbf{e}}_{n_{2}} \ldots \hat{\mathbf{e}}_{n_{k}}$ also refers to the natural product.

As an example, let us say the following natural product is to be evaluated:

$$
\left[\begin{array}{l}
a \\
b
\end{array}\right] \wp\left[\begin{array}{l}
x \\
y
\end{array}\right]
$$

First, we express each in unit vector notation:

$$
\left(a \hat{\mathbf{e}}_{1}+b \hat{\mathbf{e}}_{2}\right) \wp\left(x \hat{\mathbf{e}}_{1}+y \hat{\mathbf{e}}_{2}\right)
$$

Then we compute the natural product:

$$
\left(a \hat{\mathbf{e}}_{1}+b \hat{\mathbf{e}}_{2}\right) \wp\left(x \hat{\mathbf{e}}_{1}+y \hat{\mathbf{e}}_{2}\right)=a x \hat{\mathbf{e}}_{1}^{2}+b x \hat{\mathbf{e}}_{2} \hat{\mathbf{e}}_{1}+a y \hat{\mathbf{e}}_{1} \hat{\mathbf{e}}_{2}+b y \hat{\mathbf{e}}_{2}^{2}
$$

This is simply equal to the matrix:

$$
\left[\begin{array}{l}
a \\
b
\end{array}\right] \wp\left[\begin{array}{l}
x \\
y
\end{array}\right]=\left[\begin{array}{ll}
a x & b y \\
a y & b x
\end{array}\right]
$$

We can do this for any n-dimensional matrix or vector. For example,

$$
\left[\begin{array}{ll}
p & q \\
r & s
\end{array}\right] \wp\left[\begin{array}{l}
a \\
b
\end{array}\right]=\left(p \hat{\mathbf{e}}_{1}^{2}+q \hat{\mathbf{e}}_{2} \hat{\mathbf{e}}_{1}+r \hat{\mathbf{e}}_{2} \hat{\mathbf{e}}_{1}+s \hat{\mathbf{e}}_{2}^{2}\right) \wp\left(a \hat{\mathbf{e}}_{1}+b \hat{\mathbf{e}}_{2}\right)
$$




$$
=a p \hat{\mathbf{e}}_{1}^{3}+b p \hat{\mathbf{e}}_{1}^{2} \hat{\mathbf{e}}_{2}+a q \hat{\mathbf{e}}_{2} \hat{\mathbf{e}}_{1}^{2}+b q \hat{\mathbf{e}}_{2} \hat{\mathbf{e}}_{1} \hat{\mathbf{e}}_{2}+a r \hat{\mathbf{e}}_{1} \hat{\mathbf{e}}_{2} \hat{\mathbf{e}}_{1}+a s \hat{\mathbf{e}}_{2}^{2} \hat{\mathbf{e}}_{1}+b s \hat{\mathbf{e}}_{2}^{3}
$$

The answer is a three dimensional matrix.

\section{An Application of the Natural Product in Matrix Calculus}

The divergence of a vector field can be considered to be the dot product of the Del operator and the vector field. The curl of a vector field can be considered to be the cross product of the Del operator and the vector field. A natural question would be, what about the natural product of the Del operator and the vector field?In this section, we shall not yet make any attempt of finding out what it geometrically means but we shall go through the mechanics of doing so for a vector field with two components.

$$
\nabla_{\wp} \mathbf{f}=\left[\begin{array}{c}
\frac{\partial}{\partial x} \\
\frac{\partial}{\partial y}
\end{array}\right] \wp\left[\begin{array}{c}
P(x, y) \\
Q(x, y)
\end{array}\right]=\left[\begin{array}{cc}
\frac{\partial P}{\partial x} & \frac{\partial P}{\partial y} \\
\frac{\partial Q}{\partial x} & \frac{\partial Q}{\partial y}
\end{array}\right]
$$

This is essentially just a Jacobian matrix.

\section{Relationship to Tensor Products}

If two vectors, $\mathbf{u}$ and $\mathbf{v}$ are given such that $\mathbf{u} \in \mathbb{R}^{n}, \mathbf{v} \in \mathbb{R}^{n}$ and

$$
\mathbf{u}=\left[\begin{array}{c}
u_{1} \\
u_{2} \\
\vdots \\
u_{m}
\end{array}\right] \text { and } \mathbf{v}=\left[\begin{array}{c}
v_{1} \\
v_{2} \\
\vdots \\
v_{n}
\end{array}\right]
$$

Then the tensor product of the 2 real vectors, $\mathbf{u} \otimes \mathbf{v}$, which is really their outer product, is defined as

$$
\mathbf{u} \otimes \mathbf{v}=\left[\begin{array}{cccc}
u_{1} v_{1} & u_{1} v_{2} & \cdots & u_{1} v_{n} \\
u_{2} v_{1} & u_{2} v_{2} & \cdots & u_{2} v_{n} \\
\vdots & \vdots & \ddots & \vdots \\
u_{m} v_{1} & u_{m} v_{2} & \cdots & u_{m} v_{n}
\end{array}\right]
$$

This is the same as

$$
\mathbf{u} \otimes \mathbf{v}=(\mathbf{u} \wp \mathbf{v})^{T}
$$

So the natural product of two vectors is essentially the transpose of their tensor product. However, it is to be noted that this is generally true only for real vectors and no other matrix or clif, including complex vectors.

\section{A Geometrical Interpretation of Matrices}

From what this paper has shown, matrices can be written as a nonlinear combination of the standard basis unit vectors. We postulate the existence of the natural space, denoted by $\mathbb{M}$. Matrices live in the natural space. Anything that can be written as a linear or nonlinear combination of the standard basis unit vectors lives in the natural space. So, $\mathbb{C}^{n} \subseteq \mathbb{M}$. The natural space is not to be confused with the exterior space of Clifford Algebra.

Then, $n$ dimensional matrices have $\mathrm{n}$ directions and an $n$-dimensional hypervolume. However, this is not to be confused with tensors like bivectors, trivectors etc. Additionally, it is known from Clifford Algebra that $\mathbb{C}^{n} \subseteq \mathbb{E}$ where $\mathbb{E}$ is the Exterior Space.

Since $\mathbb{C}^{n}$ is the largest space which is a subset of both the natural space and the exterior space (i.e. if there exists some set $S$ such that $S \subseteq \mathbb{E}$ and then $S \subseteq \mathbb{M}$ ), the conclusion is drawn that $\mathbb{C}^{n}=\mathbb{M} \cap \mathbb{E}$.

\section{Acknowledgements}

The author is grateful to Dr. Bharath Kalyan, Research Associate, National University of Singapore for his valuable comments on earlier drafts.

\section{References}

Gibbs, J. (1913). Vector Analysis. New Haven: Yale University Press. 
Hamilton, W. (1866). Elements of Quaternions. London: Longmans, Green \& Co.

Latyshev, V. (2005). A general version of the standard basis and its application to T-ideals. Acta Applicandae Mathematicae, 85(1), 219-221. http://dx.doi.org/10.1007/s10440-004-5623-3 